ASC Report No. 35/2013

\title{
Density Profile Equation with $p$-Laplacian: Analysis and Numerical Simulation
}

G. Hastermann, P.M. Lima, M.L. Morgado, E.B. Weinmüller 


\section{Most recent ASC Reports}

34/2013 P. Amodio, T. Levitina, G. Settani, E.B. Weinmüller

Numerical simulation of the whispering gallery modes in prolate spheriods

33/2013 N. Zamponi and A. Jüngel

Global existence analysis for degenerate energy-transport models for semiconductors

32/2013 F. Achleitner, C.M. Cuesta, S. Hittmeir

Travelling waves for a non-local Korteweg-de Vries-Burgers equation

31/2013 T. Hohage, L. Nannen

Convergence of infinite element methods for scalar waveguide problems

30/2013 M. Feischl, T. Führer, M. Karkulik, J.M. Melenk, and

D. Praetorius

Quasi-optimal convergence rates for adaptive boundary element methods with data approximation,

Part II: Hyper-singular integral equation

29/2013 W. Auzinger, A.S. Bagherzadeh, O. Koch

Error estimation based on locally weighted defect for boundary value problems in second order ordinary differential equations

$28 / 2013$ I.G. Graham, M. Löhndorf, J.M. Melenk, E.A. Spence

When is the error in the $h$-BEM for solving the Helmholtz equation bounded independently of $k$ ?

27/2013 R. Hammer, W. Pötz, and A. Arnold

Single-cone real-space finite difference scheme for the time-dependent Dirac equation

26/2013 M. Aurada, M. Ebner, M. Feischl, S. Ferraz-Leite, T. Führer,

P. Goldenits, M. Karkulik, M. Mayr, and D. Praetorius

Hilbert (Release 3): A MATLAB implementation of adaptive BEM

25/2013 M. Feischl, T. Führer, M. Karkulik, D. Praetorius,

and E.P. Stephan

Efficient additive Schwarz preconditioning

for hypersingular integral equations

on locally refined triangulations

Institute for Analysis and Scientific Computing

Vienna University of Technology

Wiedner Hauptstraße 8-10

1040 Wien, Austria

E-Mail: admin@asc.tuwien.ac.at

WWW: http://www.asc.tuwien.ac.at

FAX: $\quad+43-1-58801-10196$

ISBN 978-3-902627-05-6

(C) Alle Rechte vorbehalten. Nachdruck nur mit Genehmigung des Autors.

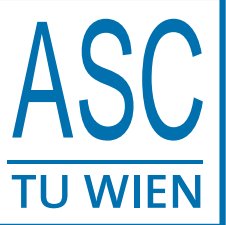




\title{
Density Profile Equation with p-Laplacian: Analysis and Numerical Simulation
}

\author{
G. Hastermann \\ Institute for Analysis and Scientific Computing, Vienna University of Technology, Austria \\ P.M. Lima \\ Centro de Matemática e Aplicações, Instituto Superior Técnico, Universidade Técnica de Lisboa, Av. Rovisco Pais, \\ 1049-001 Lisboa, Portugal \\ M.L. Morgado \\ CM-UTAD and Dep. Matematica, UTAD, Vila Real, Portugal \\ E.B. Weinmüller \\ Institute for Analysis and Scientific Computing, Vienna University of Technology, Austria
}

\begin{abstract}
Analytical properties of a nonlinear singular second order boundary value problem in ordinary differential equations posed on an unbounded domain for the density profile of the formation of microscopic bubbles in a nonhomogeneous fluid are discussed. Especially, sufficient conditions for the existence and uniqueness of solutions are derived. Two approximation methods are presented for the numerical solution of the problem, one of them utilizes the open domain Matlab code bvpsuite. The results of numerical simulations are presented and discussed.
\end{abstract}

Keywords: Singular boundary value problems, nonlinear ordinary differential equations, degenerate Laplacian, collocation methods, shooting methods.

2000 MSC: 65L05,34B16

\section{Introduction}

In this paper, we consider second order nonlinear ordinary differential equations (ODEs) arising in the modeling of non-homogeneous fluids. In the Cahn-Hilliard theory for mixtures of fluids (see, for example, [5]) an additional term involving the gradient of the density $(\operatorname{grad}(\rho))$ is added to the classical expression $E_{0}(\rho)$ for the volume free energy, depending on the density $\rho$ of the medium. Hence the total volume free energy of a non-homogeneous fluid can be written as

$$
E(\rho, \operatorname{grad}(\rho))=E_{0}(\rho)+\frac{\sigma}{2}(\operatorname{grad}(\rho))^{2},
$$

where $E_{0}(\rho)$ is a double-well potential, whose wells define the phases. The potential $E_{0}(\rho)$ causes an interfacial layer within which the density $\rho$ suffers large variations [10].

Email addresses: e-mail:gottihastermann@gmx.at (G. Hastermann), e-mail: plima@math.ist.utl.pt ( P.M. Lima), e-mail: luisam@utad.pt (M.L. Morgado), e-mail:e.weinmueller@tuwien.ac.at (E.B. Weinmüller) 
Because of the shape of $E_{0}$, the fluid tends to divide into two phases with densities $\rho=\rho_{l}$ (liquid) and $\rho=\rho_{v}$ (vapour) and the term $\frac{\sigma}{2}(\operatorname{grad}(\rho))^{2}$ tends to turn the interface between them into a thin layer, endowing it with energy (the surface tension) [26].

When the free energy is given by (1) the density profile $\rho(r)$ can be obtained in the stationary case by means of minimization of the functional,

$$
J(\rho)=\int_{\Omega}\left(E_{0}(\rho)+\frac{\sigma}{2}(\operatorname{grad}(\rho))^{2}\right) d r
$$

where $\Omega \subset R^{N}$. This minimization problem leads to a partial differential equation of the form

$$
\gamma \nabla \rho=\mu(\rho)-\mu_{0},
$$

where $\mu(\rho)=\frac{d E_{0}}{d \rho}$ is the chemical potential of the considered mixture of fluids. In the case of spherical symmetry, which is the most common in applications, equation (3) can be reduced to a second order ODE of the form

$$
r^{1-N}\left(r^{N-1} \rho^{\prime}(r)\right)^{\prime}=f(\rho(r)), \quad r>0,
$$

where $N$ is the space dimension and $f$ represents the right-hand side of (4). This function is usually known and depends on the properties of the considered mixture of fluids. Typically it is a cubic polynomial of $\rho$, with three real roots. Choosing an adequate system of units, we may write $f$ as

$$
f(\rho):=4 \lambda^{2} \rho(\rho+1)(\rho-\xi),
$$

where $\xi=\rho_{v}$ (vapour density) and $\lambda$ is a real parameter. Equation (4) is called the density profile equation and was studied, for example in $[6,7]$. The authors of those articles show that the density profile $(\rho)$ is a monotone solution of equation (4) satisfying the boundary conditions,

$$
\rho^{\prime}(0)=0, \quad \lim _{r \rightarrow \infty} \rho(r)=\xi
$$

In $[14,20,22]$, a detailed study of the boundary value problem (BVP) (4), (6) has been provided. In [20, 22] the asymptotic properties of the solutions near the singular points, 0 and $\infty$, have been studied. This enables approximate representations of the solution for $r \rightarrow 0$ and $r \rightarrow \infty$. Based on these representations, stable shooting methods were implemented for the numerical solution of the problem. Moreover, in [14], accurate numerical results were obtained for this problem, using the bvpsuite code based on polynomial collocation.

In the present paper, we consider a more general problem, where the the energy integral has the form

$$
J(\rho)=\int_{\Omega}\left(E_{0}(\rho)+\frac{c}{p}(\operatorname{grad}(\rho))^{p}\right) d r
$$

where $p>1$ is a given constant ${ }^{1}$. Such models were analyzed in [24, 25], where the authors have studied the minimization of the functional (7), which has led them to the analysis of a partial differential equation of the form

$$
\Delta_{p} u:=c \operatorname{div}\left(|\operatorname{grad}(\rho)|^{p-2} \operatorname{grad}(\rho)\right)=\frac{d E_{0}}{d \rho} .
$$

The operator $\Delta_{p}$ on the left-hand side of (8) is the so called $p$-Laplacian. This operator enables the description of mixtures of fluids of a more general nature when compared to the classical Cahn-Hilliard theory, $p=2$. In particular, with this formulation we can deal with the case where the surface tension of the fluid is not constant in the region of the interface. As for $p=2$, we consider here spherical bubbles, and therefore spherical symmetry is used to reduce the dimension of the model. The study of the general

\footnotetext{
${ }^{1}$ For $p=2$ we obtain the case $(2)$
} 
case, without spherical symmetry, can be found in [24, 25]. Using spherical coordinates and introducing a convenient system of units, the following ODE arises:

$$
r^{1-N}\left(r^{N-1}\left|\rho^{\prime}(r)\right|^{p-2} \rho^{\prime}(r)\right)^{\prime}=f_{p}(\rho), \quad r>0,
$$

where $f_{p}$ is a function which reduces to $f$, given by (5), in the case $p=2$. The form of $f_{p}$ for $p \neq 2$ will be discussed in the next section. We are interested in strictly monotone solutions of (9) satisfying the boundary conditions specified in (6).

It is worth mentioning that a closely related problem was analyzed in the recent papers $[8,27]$, where the authors consider

$$
\epsilon^{p} r^{1-N}\left(r^{N-1}\left|\rho^{\prime}(r)\right|^{p-2} \rho^{\prime}(r)\right)^{\prime}-W^{\prime}(\rho)=0, \quad r>0 .
$$

Here, the function $W$ is a double-well potential (like $E_{0}$ in our case), and $\epsilon$ is an unknown parameter which has to be determined. The solution of equation (10) has to satisfy the boundary conditions,

$$
\rho^{\prime}(0)=\rho(R)=\rho^{\prime}(R)=0,
$$

where $R$ is a given constant. Under certain conditions on $W(\rho), p$, and $N$, the existence and uniqueness of solution of the problem (6), (10) is shown in the case when $\epsilon$ belongs to a certain set of the eigenvalues $\epsilon_{n}$, $n \in \mathbf{N}$.

In the present paper we analyze the BVP (6), (9) and propose efficient numerical methods for its approximate solution.

The paper is organized as follows. In Section 2, we discuss existence and uniqueness of solutions to the BVP (6),(9). In Section 3, a shooting method based on the asymptotic properties of solutions and a collocation method are introduced. Numerical results are presented in Section 4 and conclusions can be found in Section 5 .

\section{Existence and Uniqueness of Solutions to the Analytical Problem}

In order to investigate the existence and uniqueness of the solution to problem (6), (9), we exploit the results presented in [9]. Especially, we focus on Corollary 1 [9].

We first carry out the following variable substitution in equation (9):

$$
\rho=\xi-u \text {. }
$$

Note that $\xi$ is the constant mentioned in the second formula of (6). This substitution transforms formula (9) to the form

$$
r^{1-N}\left(r^{N-1}\left|u^{\prime}(r)\right|^{p-2} u^{\prime}(r)\right)^{\prime}+g_{p}(u)=0, \quad r>0,
$$

where $g_{p}(u):=f_{p}(\xi-u)$, and the boundary conditions (6) are replaced by

$$
u^{\prime}(0)=0, \quad \lim _{r \rightarrow \infty} u(r)=0 .
$$

Below, we will use the following notation:

$$
G_{p}(s):=\int_{0}^{s} g_{p}(u) d u .
$$

Using (14) the following conditions are formulated.

C1. There exists a constant $\beta>0$ such that $G_{p}(s)<0$ for $0<s<\beta$ and $G_{p}(\beta)=0$.

C2. There exists a constant $\gamma>\beta$ such that $g_{p}(\gamma)=0$.

C3. Function $g_{p}(u) /(u-\beta)^{p-1}$ is a non-increasing for $\beta<u<\gamma$.

For our problem setting, Corollary 1 [9] reads: 
Figure 1: Graphs of the functions $g_{p}$ and $G_{p}$ for $p=3, \xi=0.5$, and $\alpha=1$.

Theorem 2.1. Assume $N \geq 2$ and $p \geq 2$.

1. If conditions $\mathbf{C 1}, \mathbf{C 2}$, and $\mathbf{C 3}$ hold, then there exists a solution $u(r)$ of the differential equation (12) and its initial value $u(0) \in(\beta, \gamma]$.

2. If additionally, $g_{p}(u)$ is a Lipschitz continuous function in the interval $(\beta, \gamma)$, then this solution is unique in the class of sufficiently smooth functions $u(r)$ with $u(0)<\gamma$.

3. The above solution is positive for all $r \geq 0$ if and only if

$$
\int_{0}^{a}\left|G_{p}(s)\right|^{-1 / p} d s=\infty
$$

for any sufficiently small $a>0$ such that $g_{p}(u) \leq 0,0 \leq u \leq a$.

We now define the inhomogeneous term $g_{p}(u)$ of (12) in such a way that all assumptions formulated in Theorem 2.1 are satisfied. First of all, according to (5),

$$
g_{2}(u)=f(\xi-u)=4 \lambda^{2}(u-\xi-1)(\xi-u) u .
$$

Moreover, since $g_{p}$ and $g_{2}$ must have the same zeros and the same sign, for any value of $p$, we search for $g_{p}$ of the form

$$
g_{p}(u)=2 p \lambda^{2}(u-\xi-1)(\xi-u) u|u-\xi-1|^{\alpha}|u|^{\alpha},
$$

where $p \geq 2$ and $\alpha \geq 0$ are adjustable real parameters. Note that the right-hand side of the classical equation is obtained by setting $\alpha=0$ and $p=2$ in (16).

It follows from the definition of $G_{p}(s)$ that this function has two real zeros denoted further as $s_{1}, s_{2}$ and such that $\xi<s_{1}<\xi+1<s_{2}$ for any $\xi$ satisfying the condition $0<\xi<1$. Therefore, condition C1 holds with the parameter $\beta=s_{1}$ where $s_{1}$ is the least positive root of the equation $G_{p}(s)=0$. Condition C2 is also satisfied for $\gamma=\xi+1$ (see, for instance, Figure 1). Concerning condition C3, we know that the function $g_{p}(u)$ attains a maximum at a certain $u_{0} \in[\beta, \gamma]$ (see the above figure). For $u \in\left[u_{0}, \gamma\right], g_{p}(u)$ is non-increasing, and so is the function $h(u):=g_{p}(u) /(u-\beta)^{p-1}$. To study the behavior of $h(u)$ in the interval $\left[\beta, u_{0}\right]$, we consider its first derivative

$$
h^{\prime}(u)=\frac{g_{p}^{\prime}(u)(u-\beta)^{p-1}-g_{p}(u)(p-1)(u-\beta)^{p-2}}{(u-\beta)^{2 p-2}} .
$$

Using the mean value theorem, we conclude that, for any $u \in\left[\beta, u_{0}\right]$, there exists some $\eta \in[\beta, u]$ such that

$$
g_{p}(u)-g_{p}(\beta)=g_{p}^{\prime}(\eta)(u-\beta)
$$

Moreover, since $g_{p}(\beta)>0$ and $p \geq 2$, we arrive at

$$
g_{p}(u)(p-1)>g_{p}(u)>g_{p}(u)-g_{p}(\beta) .
$$


It follows from (18) and (19) that

$$
g_{p}(u)(p-1)(u-\beta)^{p-2}>g_{p}^{\prime}(\eta)(u-\beta)^{p-1}
$$

for some $\eta \in[\beta, u]$. Also, it is clear that $g_{p}^{\prime}(\eta) \geq g_{p}^{\prime}(u)$ since $g_{p}^{\prime \prime}(u) \leq 0$ in $[\beta, u]$. Taking formula (20) into account, we conclude from $(17)$ that $h^{\prime}(u) \leq 0$, and, hence, condition C3 holds.

It follows from formula (16) that the function $g_{p}(u)$ is Lipschitz continuous in the interval $(\beta, \gamma)$ and therefore the uniqueness (see Assertion 2 in Theorem 2.1) follows.

Next, the integral $G_{p}(s)$ can be represented in the form

$$
G_{p}(s)=-\lambda^{2} s^{2}|s|^{\alpha} P(s),
$$

where $\lambda$ is a constant and $P(s)$ is a smooth function such that $P(0) \neq 0$. This means that the function $P(s)$ has only zeros at $s_{1}$ and $s_{2}$. So the formula (21) yields

$$
\left|G_{p}(s)\right|^{-1 / p}=|\lambda|^{-2 / p}|s|^{-(\alpha+2) / p}|P(s)|^{-1 / p} .
$$

The latter formula results in the conclusion that the integral on the left-hand side of (15) diverges when $\alpha \geq p-2$ and converges for all other values of $\alpha$. Hence, for $\alpha \geq p-2$, the condition (15) is satisfied.

Finally, Theorem 2.1 states that, for the function $g_{p}(u)$ defined by $(16), \alpha \geq p-2$ and $0<\xi<1$, there exists a unique monotonously decreasing positive solution $u(r)$ to the BVP (12), (13) in case that $p \geq 2$.

Now we recall that the unknown solution of the BVP (6), (9) is $\rho=\xi-u$. So, having applied the above result and utilized the back substitution, we arrive at the following form of the inhomogeneous term in the differential equation:

$$
f_{p}(\rho)=2 p \lambda^{2}(\rho-\xi)(\rho+1) \rho|\rho-\xi|^{\alpha}|\rho+1|^{\alpha},
$$

where $\xi$ and $\alpha$ satisfy conditions mentioned above. Formula (22) ensures that the BVP (6), (9) has a unique solution for $p \geq 2$. Moreover, we choose $\alpha:=p-2$.

The second statement of Theorem 2.1 implies that $\rho(0)>-1$. The strict monotonicity of the solution and the condition $\lim _{r \rightarrow \infty} \rho(r)=\xi$ result in $-1<\rho(r)<\xi$ for any $r>0$. This means that the function $f_{p}(\rho)$ in the BVP $(6),(9)$ (see formula $(22)$ ) can be simplified to

$$
f_{p}(\rho)=-2 p \lambda^{2}(\xi-\rho)^{p-1} \rho(\rho+1)^{p-1} .
$$

Thus, we have shown that the differential equation under consideration possesses a unique solution $\rho(r)$ for any $p \geq 2$ when its inhomogeneous term $f_{p}(\rho)$ is given by $(23)$. A similar result for $p \in(1,2)$ remains an open issue.

Note that the existence conditions for problem (6), (9) require $\xi<1$, which means that the function $f_{p}$, defined by (23), cannot be an odd function. This is not the case for the eigenvalue problem (10), (11), where solutions exist in the case when $W$ is an even function (thus $W^{\prime}$ is odd).

\section{Numerical Approximation}

\subsection{Shooting Method}

In order to design a suitable shooting method it was necessary to accordingly extend the algorithm described in [22]. The main idea is to replace the considered BVP with two singularities, at the origin and at infinity, by two auxiliary BVPs, each of them having only one singularity. Since results presented in Section 4 were obtained using the shooting method, we give here an outline of the algorithm. Further details can be found in $[21]$.

Step 1. We start by fixing the values of $r_{0}, \delta$, and $r_{\infty}$, in such a way that $r_{\infty}>r_{0}>\delta>0$. Here, $r_{0}$ is an initial approximation to the root of the solution, the so-called bubble radius, $\delta$ is close to zero and $r_{\infty}$ is sufficiently large for a reasonable approximation of the solution at infinity, obtained in [21]. Next, we subdivide the region $\left[\delta, r_{\infty}\right]$ in which the approximate solution will be provided into two subintervals, $\left[\delta, r_{0}\right]$ and $\left[r_{0}, r_{\infty}\right]$. We denote by $\rho_{-}(r)$ and $\rho_{+}(r)$ monotone solutions of $(9)$ on $\left[\delta, r_{0}\right]$ and $\left[r_{0}, r_{\infty}\right]$, respectively. 
The first of these functions satisfies $\rho_{-}\left(r_{0}\right)=0$ and a boundary condition at $\delta$, which follows from the asymptotic behavior of the solution at the origin. The second one satisfies $\rho_{+}\left(r_{0}\right)=0$ and a condition at $r_{\infty}$, which reflects the asymptotic behavior of the solution at infinity. Each of these functions can be computed as the solution of a regular BVP using a standard shooting method. Then we define

$$
\rho(r)=\left\{\begin{array}{l}
\rho_{-}(r), \text { if } \delta \leq r \leq r_{0}, \\
\rho_{+}(r), \text { if } r_{0} \leq r \leq r_{\infty}
\end{array}\right.
$$

Step 2. In general, the function $\rho(r)$ from $(24)$ is not a solution of $(6),(9)$ on $[0, \infty[$ because the condition

$$
\lim _{r \rightarrow r_{0}-} \rho^{\prime}(r)=\lim _{r \rightarrow r_{0}+} \rho^{\prime}(r)
$$

is not satisfied for the given $r_{0}$. Therefore we compute the difference

$$
\Delta\left(r_{0}\right)=\lim _{r \rightarrow r_{0}-} \rho^{\prime}(r)-\lim _{r \rightarrow r_{0}+} \rho^{\prime}(r) .
$$

Our goal is now to find such a value $r_{0} \in \mathbb{R}_{+}$that $\Delta\left(r_{0}\right)=0$. In order to find the required value of $r_{0}$ we use again the shooting method.

Step 3. In order to extend the approximate solution to the intervals $[0, \delta]$ and $\left[r_{\infty}, \infty\right)$, we use the asymptotic expansions derived in [21].

The method has been implemented using MATHEMATICA. This enables to compute the approximations accurately within a reasonable computational time for a large range of values $p$ and $\xi$. The advantage of the proposed approach is that it makes use of the asymptotic solution properties near the singular points. In the present implementation, the auxiliary problems for $\rho_{-}$and $\rho_{+}$are solved using the package NDSOLVE in Mathematica [28]. The drawback of this method is that one can neither control the global error of the approximation nor the required total computational effort. In Section 3.2, we describe a different approach to the numerical solution of the problem (6), (9). This approach is based on polynomial collocation and makes use of the open domain MATLAB code bvpsuite, cf. [15], designed for the numerical solution of BVPs in ODEs, posed on unbounded domain.

\subsection{Collocation Method}

The code bvpsuite is designed to solve systems of differential equations of arbitrary mixed order including zero $^{2}$, subject to initial or boundary conditions,

$$
\begin{gathered}
F\left(t, p_{1}, \ldots, p_{s}, z_{1}(t), z_{1}^{\prime}(t), \ldots, z_{1}^{\left(l_{1}\right)}(t), \ldots, z_{n}(t), z_{n}^{\prime}(t), \ldots, z_{n}^{\left(l_{n}\right)}(t)\right)=0 \\
B\left(p_{1}, \ldots, p_{s}, z_{1}\left(c_{1}\right), \ldots, z_{1}^{\left(l_{1}-1\right)}\left(c_{1}\right), \ldots, z_{n}\left(c_{1}\right), \ldots z_{n}^{\left(l_{n}-1\right)}\left(c_{1}\right), \ldots, z_{1}\left(c_{q}\right), \ldots,\right. \\
\left.z_{1}^{\left(l_{1}-1\right)}\left(c_{q}\right), \ldots, z_{n}\left(c_{q}\right), \ldots, z_{n}^{\left(l_{n}-1\right)}\left(c_{q}\right)\right)=0
\end{gathered}
$$

where the solution $z(t)=\left(z_{1}(t), z_{2}(t), \ldots, z_{n}(t)\right)^{T}$, and the parameters $p_{i}, i=1, \ldots, s$, are unknown. In general, $t \in[a, b], 0 \leq a, b<\infty^{3}$. Moreover, $F:[a, b] \times \mathbb{R}^{s} \times \mathbb{R}^{l_{1}+1} \times \cdots \times \mathbb{R}^{l_{n}+1} \rightarrow \mathbb{R}^{n}$ and $B$ : $\mathbf{R}^{s} \times \mathbf{R}^{q l_{1}} \times \cdots \times \mathbf{R}^{q l_{n}} \rightarrow \mathbf{R}^{l+s}$, where $l:=\sum_{i=1}^{n} l_{i}$. Note that boundary conditions can be posed on any subset of distinct points $c_{i} \in[a, b]$, with $a \leq c_{1}<c_{2}<\cdots<c_{q} \leq b$. For the numerical treatment, we assume that BVP (25) is well-posed and has a locally unique solution $z$.

\footnotetext{
${ }^{2}$ This means that differential-algebraic equations are also in the scope of the code.

${ }^{3}$ For the extension to unbounded domains, see Section 3.2.1.
} 
In order to find a numerical solution of $(25)$ we consider a mesh $\left[\tau_{i}, \tau_{i+1}\right], i=0, \ldots, N-1$, partitioning the interval $[a, b]$. Every subinterval $\left[\tau_{i}, \tau_{i+1}\right]$ contains $m$ collocation points $t_{i, j}, j=1, \ldots, m$. Let $\mathbf{P}_{m}$ be the space of piecewise polynomial functions of degree $\leq m$, which are globally continuous in $[a, b]$. In every subinterval $J_{i}$ we make an ansatz $P_{i, k} \in \mathbf{P}_{m+l_{k}-1}$ for the $k$-th solution component $z_{k}, k=1, \ldots, n$, of the problem (25). In order to compute the coefficients in the ansatz functions we require that (25) is satisfied exactly at the collocation points. Moreover we require that the collocation polynomial $p(t):=P_{i}(t), t \in J_{i}$, is a globally continuous function on $[a, b]$ with components in $C^{l_{i}-1}[a, b], i=1, \ldots, n$, and that the boundary conditions hold. All these conditions imply a nonlinear system of equations for the unknown coefficients in the ansatz function. For more details see $[15,16]$.

\subsubsection{Semi-Infinite Intervals}

Our code can also treat problems posed on semi-infinite intervals $\tau \in[a, \infty), a>0$ (and by a splitting of the interval, also $a=0$ ). In order to exploit the mesh selection strategy which works efficiently on finite intervals, we transform the unbounded domain to a finite one. Let us explain the transformation using the simple problem class

$$
x^{\prime}(\tau)=\tau^{\beta} F(\tau, x(\tau)), \tau \in[a, \infty), a>0, \beta>-1 .
$$

By introducing $t:=\frac{1}{\tau}, z(t):=x\left(\frac{1}{\tau}\right)$, we obtain a new system for $z$,

$$
z^{\prime}(t)=-F(1 / t, z(t)) / t^{\beta+2}, t \in(0,1 / a], \beta+2>1 .
$$

This is in general a problem with an essential singularity, which however is in the scope for the collocation method, error estimation procedure and adaptive mesh refinement. In this approach, the mesh is adapted only according to the unsmoothness of the solution without the need for mesh grading on long intervals, and moreover no truncation of the unbounded interval is necessary. This strategy was employed successfully for example in $[4,14]$.

\subsubsection{Reformulation of the Original Problem for the Collocation Method}

We now solve the problem (6), (9) using the collocation method implemented in bvpsuite. Here, the original differential equation is transformed to a system of four implicit first order differential equations. The main idea is to split the unbounded domain $(0, \infty)$ the original differential equation is posed on, into $(0,1)$ and $[1, \infty)$.

For $r \in(0,1)$, we define the independent and dependent variables as $t:=r$ and $z_{1}(t):=\rho(r)$, respectively. Let us introduce

$$
z_{3}(t):=\left|z_{1}^{\prime}(t)\right|^{p-2} z_{1}^{\prime}(t)
$$

Then, for $z_{3} \in C^{1}(0,1)$, it follows immediately from (9),

$$
z_{3}^{\prime}(t)+\frac{N-1}{t} z_{3}(t)=f_{p}\left(z_{1}(t)\right)
$$

For $r \in[1, \infty)$ the new variables are $t:=\frac{1}{r}$ and $z_{2}(t):=\rho\left(\frac{1}{r}\right)$. Note that $\frac{d}{d r}=\frac{d}{d t}\left(-t^{2}\right), \frac{d}{d t}=\frac{d}{d r}\left(-\frac{1}{t^{2}}\right)$ and

$$
\frac{d z_{2}}{d t}(t)=\frac{d \rho}{d r}(r)\left(-\frac{1}{t^{2}}\right) \text {. }
$$

Using (26), we rewrite (9) and obtain

$$
t^{N-1} \frac{d}{d r}\left(t^{1-N}\left|\frac{d z_{2}}{d t}(t)\left(-t^{2}\right)\right|^{p-2} \frac{d z_{2}}{d t}(t)\left(-t^{2}\right)\right)=f_{p}\left(z_{2}(t)\right), \quad t \in(0,1) .
$$

Introducing $z_{4}(t):=\left|\frac{d z_{2}}{d t}(t)\right|^{p-2} \frac{d z_{2}}{d t}(t)$ and assuming $z_{4} \in C^{1}(0,1]$, equation (27) can be written in the following form:

$$
t^{N-1} \frac{d}{d r}\left(-t^{2 p-N-1} z_{4}(t)\right)=t^{N-1} \frac{d}{d t}\left(-t^{2 p-N-1} z_{4}(t)\right)\left(-t^{2}\right)=f_{p}\left(z_{2}(t)\right) .
$$


This yields the following system of four implicit differential equations posed on $(0,1]$,

$$
\begin{aligned}
& (2 p-N-1) t^{2 p-1} z_{4}(t)+t^{2 p} z_{4}^{\prime}(t)=f_{p}\left(z_{2}(t)\right), \\
& z_{4}(t)=\left|z_{2}^{\prime}(t)\right|^{p-2} z_{2}^{\prime}(t) \\
& z_{3}^{\prime}(t)+\frac{N-1}{t} z_{3}(t)=f_{p}\left(z_{1}(t)\right) \\
& z_{3}(t)=\left|z_{1}^{\prime}(t)\right|^{p-2} z_{1}^{\prime}(t) .
\end{aligned}
$$

For the above system four boundary conditions are required. From (6) we have

$$
z_{3}(0)=0, \quad z_{2}(0)=\xi
$$

Furthermore, both solution branches $z_{1}$ and $z_{2}$ shall match in $t=1$ in such a way that $\rho \in C^{1}(0, \infty)$ holds. Consequently, again using (26), we require,

$$
z_{1}(1)=z_{2}(1), \quad z_{3}(1)=-z_{4}(1)
$$

\section{Numerical Results}

In this section, we present numerical results obtained by the shooting and collocation methods described above. The main purpose of the numerical simulation is to test and compare the efficiency and robustness of the proposed algorithms.

We begin by presenting numerical results which show how the physical properties of the bubbles depend on $p$ and $\xi$. Besides the bubble radius $R$ and the gas density at the center of the bubble, we will also calculate the numerical values of the energy integral $J$ defined by

$$
J:=J(\rho):=\int_{0}^{\infty}\left(\frac{\rho^{\prime}(r)^{p}}{p}+W_{p}(\rho(r))\right) r^{N-1} d r, \quad W_{p}(\rho)=\int_{0}^{\rho} g_{p}(u) d u .
$$

The solution of the BVP can be viewed as the function $\rho$ for which the integral $J$ is minimized.

In order to evaluate $J$, we use numerical quadrature.

Keeping in mind that we use a collocation method to solve (29)-(32), collocation points could be used as mesh points in the involved quadrature rule. We now introduce the partition $\Delta_{\mathcal{T}}$ of the interval of integration $[0,1], \Delta_{\mathcal{T}}=\left\{0=\tau_{0}<\tau_{1}, \ldots, \tau_{j}<\tau_{j+1}, \ldots, \tau_{N}=1,\right\}$ with $h_{j}:=\tau_{j+1}-\tau_{j}$. In each subinterval $\left[\tau_{j}, \tau_{j+1}\right]$, we specify $m$ collocation points $t_{j, l}, l=1,2, \ldots, m$, such that $\tau_{j}<t_{j, 1}<t_{j, 2}<\ldots<t_{j, m}<\tau_{j+1}$.

Let us denote by $P_{j, k}(t)$ the collocation polynomial approximating $z_{k}(t)$ for $t \in\left[\tau_{j}, \tau_{j+1}\right], k=1, \ldots, 4$, $j=0,1, \ldots, N-1$. Then, we can approximate the values of the first derivative $z_{k}^{\prime}(t)$ at the collocation points $t_{j, l}, j=0,1, \ldots, N-1, l=1,2, \ldots, m$, directly from the definition of the collocation method. Since $z_{1}$ is monotonically increasing, we conclude from (32),

$$
z_{1}^{\prime}(t)=z_{3}(t)^{\frac{1}{p-1}}
$$

and consequently,

$$
P_{j, 1}^{\prime}\left(t_{j, l}\right)=P_{j, 3}\left(t_{j, l}\right)^{\frac{1}{p-1}},
$$

$j=0,1, \ldots, N-1, l=1,2, \ldots, m$. For the second component we obtain a very similar relation. Due to (26), $z_{2}$ is monotonically decreasing, and thus from (30) it follows $\left|z_{2}^{\prime}(t)\right|=z_{4}(t)\left|z_{4}(t)\right|^{\frac{1}{p-1}}$ which together with $\left|z_{2}^{\prime}\right|=-z_{2}^{\prime}$ yields

$$
\left(-P_{j, 2}^{\prime}\left(t_{j, l}\right)\right)^{p}=\left|P_{j, 4}\left(t_{j, l}\right)\right|^{\frac{p}{p-1}},
$$


$j=0,1, \ldots, N-1, l=1,2, \ldots, m$. Let us assume that a numerical quadrature rule is defined on the interval $[0,1]$ via the weights $\left\{w_{l}\right\}_{l \in\{1, \ldots, n\}}$ and the evaluation points $\xi_{l} \in[0,1], l=1, \ldots, n$,

$$
\int_{0}^{1} f(t) d t \approx Q_{[0,1]}(f)=\sum_{l=1}^{n} w_{l} f\left(\xi_{l}\right)
$$

In order to integrate over the interval $\left[\tau_{j}, \tau_{j+1}\right]$, we have to recalculate the weights and the evaluation points accordingly,

$$
\int_{\tau_{j}}^{\tau_{j+1}} f(t) d t \approx Q_{\left[\tau_{j}, \tau_{j+1}\right]}(f)=h_{j} \sum_{l=1}^{n} w_{l} f\left(\left(\tau_{j+1}-\tau_{j}\right) \xi_{l}+\tau_{j}\right)
$$

We first rewrite $J$,

$$
\begin{gathered}
J(\rho)=\int_{0}^{1}\left(\frac{1}{p} \rho^{\prime}(r)^{p}+W_{p}(\rho(r))\right) r^{N-1} d r+\int_{1}^{\infty}\left(\frac{1}{p} \rho^{\prime}(r)^{p}+W_{p}(\rho(r))\right) r^{N-1} d r= \\
\int_{0}^{1}\left(\frac{1}{p} z_{1}^{\prime}(t)^{p}+W_{p}\left(z_{1}(t)\right)\right) t^{N-1} d t+\int_{0}^{1}\left(\frac{1}{p} \frac{\left(-z_{2}^{\prime}(t)\right)^{p}}{t^{N+1+2 p}}+\frac{W_{p}\left(z_{2}(t)\right)}{t^{N+1}}\right) d t .
\end{gathered}
$$

Choosing a Gaussian quadrature rule and the collocation scheme with $m=n$ and $\left(\tau_{j+1}-\tau_{j}\right) \xi_{l}+\tau_{j}=t_{j, l}$ enables to use (33) and (34). Thus, we obtain

$$
J(\rho) \approx \sum_{\substack{j \in\{0 \ldots N-1\}, l \in\{1 \ldots m\}}} h_{j} w_{l}\left(\frac{\mid P_{j, 4}\left(t_{j, l}\right)^{\frac{p}{p-1}}}{p t_{j, l}^{N+1+2 p}}+\frac{W_{p}\left(P_{j, 2}\left(t_{j, l}\right)\right)}{t_{j, l}^{N+1}}+\left(\frac{P_{j, 3}\left(t_{j, l}\right)^{\frac{p}{p-1}}}{p}+W_{p}\left(P_{j, 1}\left(t_{j, l}\right)\right)\right) t_{j, l}^{N-1}\right)
$$

Note that in the last sum only values of the solution at the collocation points provided by bvpsuite occur and therefore the evaluation of $J$ is straightforward.

Let us denote $\rho_{0}:=\lim _{r \rightarrow 0+} \rho(r)$. This value, whose physical meaning is the gas density at the center of the bubble, is one of the important parameters that we can obtain from the numerical solution of the density profile equation.

Unfortunately, we cannot compare shooting and collocation directly, by solving the system (29)-(32) by the shooting method. In order to be able to utilize this approach, we need a very precise knowledge on the solution asymptotics. For the components $z_{1}$ to $z_{4}$ in the system (29)-(32) such information is not completely available. Therefore, we compare both methods indirectly via the values $\rho_{0}, R$ and $J$.

In Tables 1, 2, and 3, we present the numerical values of $J, R$, and $\rho_{0}$ for $p=3, p=3.5$, and $p=4$, respectively. We also give an error estimate for the approximation of $J$. The results reported in the tables are provided for the case $N=3$ and $\xi=0.1,0.2, \ldots, 0.8$. They were obtained using bvpsuite based on polynomial collocation. The application of the code is in no way straightforward and it takes quite an effort to calculate the solution with reasonable accuracy. The reason for the difficulties can be explained by the fact that the analytical solution is unsmooth and that condition numbers of the involved linear systems of equations arising during the Newton iteration become significant for $h \rightarrow 0$. This means that the starting values for the Newton procedure have to be very accurate. Clearly, we can use the solution profiles obtained for a certain value of $\xi$ as a starting guess for the next value $\xi+\Delta_{\xi}$, but for the Newton method to work $\Delta_{\xi}$ has to be small. Moreover, to recover correctly the solution behavior in the region where its profile is very steep, very fine grids are necessary. This results in high demand for computational recourses (storage and time).

In Table 4, we illustrate the convergence order of the collocation method and the condition numbers of the matrices arising during the Newton iteration. These results show clearly that the problem is ill-conditioned and a drop of the convergence order can be observed, due to the unsmoothness of the solution at the origin. 


\begin{tabular}{cccccc}
\hline$\xi$ & $J$ & $e r r_{J}$ & $\rho_{0}$ & $R$ & $e r r_{\text {sol }}$ \\
\hline 0.1 & 0.000840 & $5.25 \mathrm{e}-8$ & -0.155300 & 2.112854 & $1.92 \mathrm{e}-5$ \\
0.2 & 0.007943 & $4.69 \mathrm{e}-7$ & -0.319620 & 1.821747 & $3.45 \mathrm{e}-5$ \\
0.3 & 0.032456 & $2.15 \mathrm{e}-6$ & -0.489921 & 1.763265 & $5.58 \mathrm{e}-5$ \\
0.4 & 0.096506 & $8.96 \mathrm{e}-6$ & -0.658872 & 1.824867 & $8.77 \mathrm{e}-5$ \\
0.5 & 0.249720 & $3.75 \mathrm{e}-5$ & -0.765003 & 2.030396 & $2.20 \mathrm{e}-4$ \\
0.6 & 0.620586 & $2.17 \mathrm{e}-4$ & -0.925900 & 2.360813 & $7.87 \mathrm{e}-4$ \\
0.7 & 1.624151 & $1.72 \mathrm{e}-3$ & -0.985087 & 3.031888 & $4.22 \mathrm{e}-3$ \\
0.8 & 5.104436 & $2.75 \mathrm{e}-2$ & -0.999475 & 4.494991 & $4.29 \mathrm{e}-2$ \\
\hline
\end{tabular}

Table 1: Numerical results for $p=3$.

\begin{tabular}{cccccc}
\hline$\xi$ & $J$ & $e r r_{J}$ & $\rho_{0}$ & $R$ & $e r r_{\text {sol }}$ \\
\hline 0.1 & 0.0001225 & $2.73 \mathrm{e}-9$ & -0.125369 & 1.731092 & $1.04 \mathrm{e}-5$ \\
0.2 & 0.0018308 & $3.56 \mathrm{e}-8$ & -0.262216 & 1.534213 & $1.58 \mathrm{e}-5$ \\
0.3 & 0.0097973 & $2.43 \mathrm{e}-7$ & -0.410427 & 1.501617 & $2.22 \mathrm{e}-5$ \\
0.4 & 0.0352165 & $1.38 \mathrm{e}-6$ & -0.567779 & 1.557476 & $3.77 \mathrm{e}-5$ \\
0.5 & 0.1050056 & $7.75 \mathrm{e}-6$ & -0.726542 & 1.705349 & $7.27 \mathrm{e}-5$ \\
0.6 & 0.2916065 & $4.92 \mathrm{e}-5$ & -0.867981 & 1.991735 & $2.93 \mathrm{e}-4$ \\
0.7 & 0.8328526 & $4.28 \mathrm{e}-4$ & -0.963051 & 2.538646 & $1.74 \mathrm{e}-3$ \\
0.8 & 2.8107513 & $7.30 \mathrm{e}-3$ & -0.997383 & 3.727937 & $1.90 \mathrm{e}-2$ \\
\hline
\end{tabular}

Table 2: Numerical results for $p=3.5$.

\begin{tabular}{cccccc}
\hline$\xi$ & $J$ & $e r r_{J}$ & $\rho_{0}$ & $R$ & $e r r_{\text {sol }}$ \\
\hline 0.1 & 0.000020 & $1.01 \mathrm{e}-10$ & -0.105232 & 1.452701 & $1.23 \mathrm{e}-5$ \\
0.2 & 0.000461 & $1.30 \mathrm{e}-9$ & -0.222393 & 1.316793 & $1.63 \mathrm{e}-5$ \\
0.3 & 0.003193 & $4.20 \mathrm{e}-9$ & -0.352719 & 1.302351 & $2.01 \mathrm{e}-5$ \\
0.4 & 0.013792 & $1.29 \mathrm{e}-7$ & -0.496736 & 1.355994 & $2.84 \mathrm{e}-5$ \\
0.5 & 0.047318 & $1.36 \mathrm{e}-6$ & -0.651939 & 1.482700 & $4.27 \mathrm{e}-5$ \\
0.6 & 0.146713 & $1.18 \mathrm{e}-5$ & -0.806737 & 1.722800 & $7.21 \mathrm{e}-5$ \\
0.7 & 0.457544 & $1.22 \mathrm{e}-4$ & -0.931945 & 2.181964 & $5.69 \mathrm{e}-4$ \\
0.8 & 1.659299 & $2.31 \mathrm{e}-3$ & -0.992361 & 3.181392 & $8.53 \mathrm{e}-3$ \\
\hline
\end{tabular}

Table 3: Numerical results for $p=4$. 
In case of an appropriately smooth solution, the convergence order would be two, but in the present case the second derivative of the solution is unbounded as $r \rightarrow 0$ for $p>0$, cf. [21].

It is interesting to see that for a fixed value of $\xi$, the condition numbers of the matrices grow as $p$ decreases, which can be explained by the fact that for smaller $p$ the interior layer of the solution near the bubble radius becomes steeper. On the other hand, if we fix $p$, the condition numbers grow as $\xi$ tend to 1 . This is well illustrated by the graphs of the solutions in Figure 3. Note the special case $p=2$ [14]: Here, although the interior layer is steeper when compared with larger values of $p$, the accuracy of the results is satisfactory since in this case the solution is smooth at the origin, see Figure 3.

\begin{tabular}{cccccccc}
\hline$h$ & err $_{z 1}$ & ord & err $_{z 2}$ & ord & $\rho_{0}$ & $R$ & cond \\
\hline $2^{-3}$ & $7.6 \mathrm{e}-2$ & 2.4 & $8.2 \mathrm{e}-2$ & 2.5 & -0.82852 & 2.15258 & $5.4 \mathrm{e} 6$ \\
$2^{-4}$ & $1.5 \mathrm{e}-2$ & 2.1 & $1.5 \mathrm{e}-2$ & 2.1 & -0.77868 & 2.05249 & $1.6 \mathrm{e} 7$ \\
$2^{-5}$ & $3.5 \mathrm{e}-3$ & 2.0 & $3.6 \mathrm{e}-3$ & 2.0 & -0.76821 & 2.03516 & $1.6 \mathrm{e} 7$ \\
$2^{-6}$ & $8.6 \mathrm{e}-4$ & 2.0 & $8.8 \mathrm{e}-4$ & 2.0 & -0.76565 & 2.03124 & $1.5 \mathrm{e} 8$ \\
$2^{-7}$ & $2.2 \mathrm{e}-4$ & 2.0 & $2.2 \mathrm{e}-4$ & 2.0 & -0.76500 & 2.03040 & $1.1 \mathrm{e} 9$ \\
$2^{-8}$ & $5.4 \mathrm{e}-5$ & 2.0 & $5.5 \mathrm{e}-5$ & 2.0 & -0.76484 & 2.03013 & $1.2 \mathrm{e} 10$ \\
$2^{-9}$ & $1.4 \mathrm{e}-5$ & - & $1.4 \mathrm{e}-5$ & - & -0.76480 & 2.03006 & $1.9 \mathrm{e} 11$ \\
\hline
\end{tabular}

Table 4: Numerical results for $(p, \xi)=(3,0.5)$. Here, $\operatorname{err}_{\rho}$ is an error estimate for the absolute global error of the collocation solution, ord is an estimate of the global convergence order, and cond is the matrix condition number.

Next we present results obtained by the shooting approach. In Table 5, we show the values of the bubble radius $R$ for different values of $p$ and $\xi$. The corresponding values of $\rho_{0}$ can be found in Table 6 . In the computations by the shooting method we used values of $\delta$ between $\delta=0.001$, for the smallest value of $p$ and $\delta=0.02$, for the largest value of $p$. The value of $r_{\infty}$ was set to 6 . In the tables, for the entries denoted with $(*)$ no reliable results could be provided.

All computations with bvpsuite were carried out with the MATLAB precision of 16 digits. However, due to the ill-posedness of the problem, in some cases, especially for $\xi$ close to 1 , the results show no more than four correct digits. For the shooting method the global error can be only estimated by comparing it to the results obtained by collocation.

\begin{tabular}{cccccc}
\hline$\xi / p$ & 2.0 & 2.5 & 3.0 & 3.5 & 4.0 \\
\hline 0.2 & 2.685 & 2.20455 & 1.82180 & 1.53429 & $(*)$ \\
0.3 & 2.582 & 2.11351 & 1.76328 & 1.50167 & 1.30245 \\
0.4 & 2.721 & 2.19287 & 1.82478 & 1.55751 & 1.35611 \\
0.5 & 3.068 & 2.43240 & 2.0062 & 1.70529 & 1.48283 \\
0.6 & 3.696 & 2.88981 & 2.35947 & 1.99133 & 1.72289 \\
0.7 & 4.833 & 3.73350 & 3.02514 & 2.53590 & 2.18120 \\
\hline
\end{tabular}

Table 5: Approximations of $R$ provided by the shooting method.

\begin{tabular}{cccccc}
\hline$\xi / p$ & 2.0 & 2.5 & 3.0 & 3.5 & 4.0 \\
\hline 0.2 & -0.5681 & -0.40926 & -0.31950 & -0.26175 & $\left({ }^{*}\right)$ \\
0.3 & -0.7707 & -0.60412 & -0.48981 & -0.40913 & -0.35048 \\
0.4 & -0.90313 & -0.77370 & -0.65871 & -0.56697 & -0.49405 \\
0.5 & -0.97112 & -0.89977 & -0.81141 & -0.72578 & -0.64933 \\
0.6 & -0.99531 & -0.97121 & -0.92550 & -0.86734 & -0.80479 \\
0.7 & -0.99979 & -0.99646 & -0.98392 & -0.96266 & -0.93096 \\
\hline
\end{tabular}

Table 6: Approximations of $\rho_{0}$ provided by the shooting method.

We know from the previous results for $p=2$, see [20,22], that the bubble radius increases for $\xi \rightarrow 0$ and 

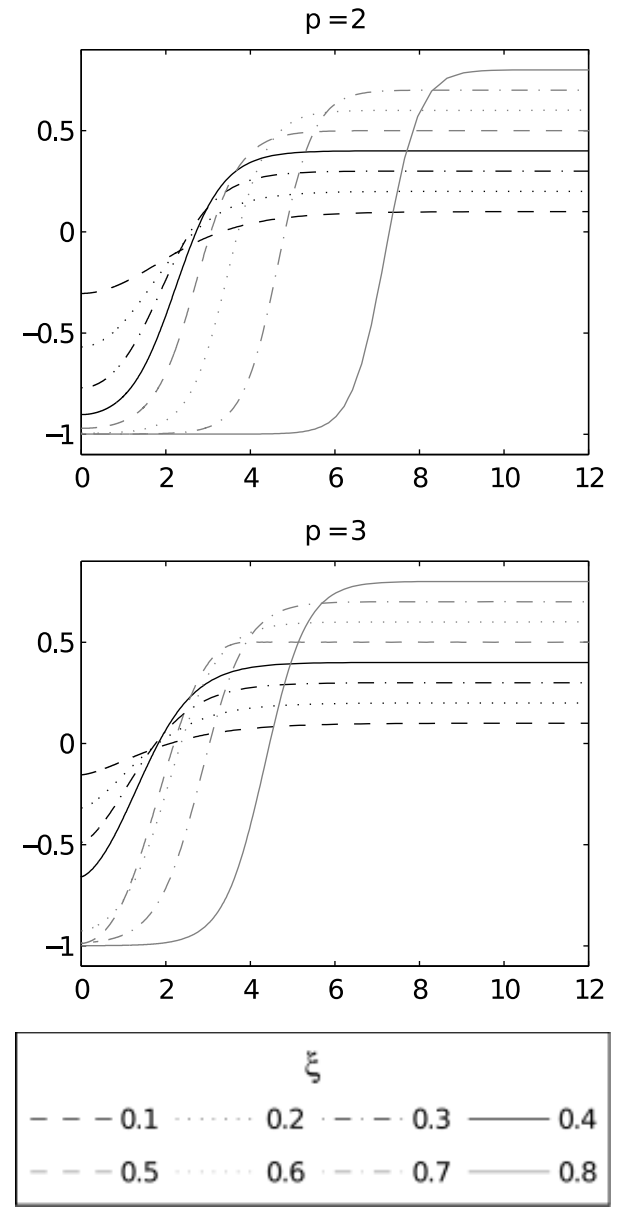

Figure 2: Solution graphs, $\rho(r)$, for different values of $p$. 

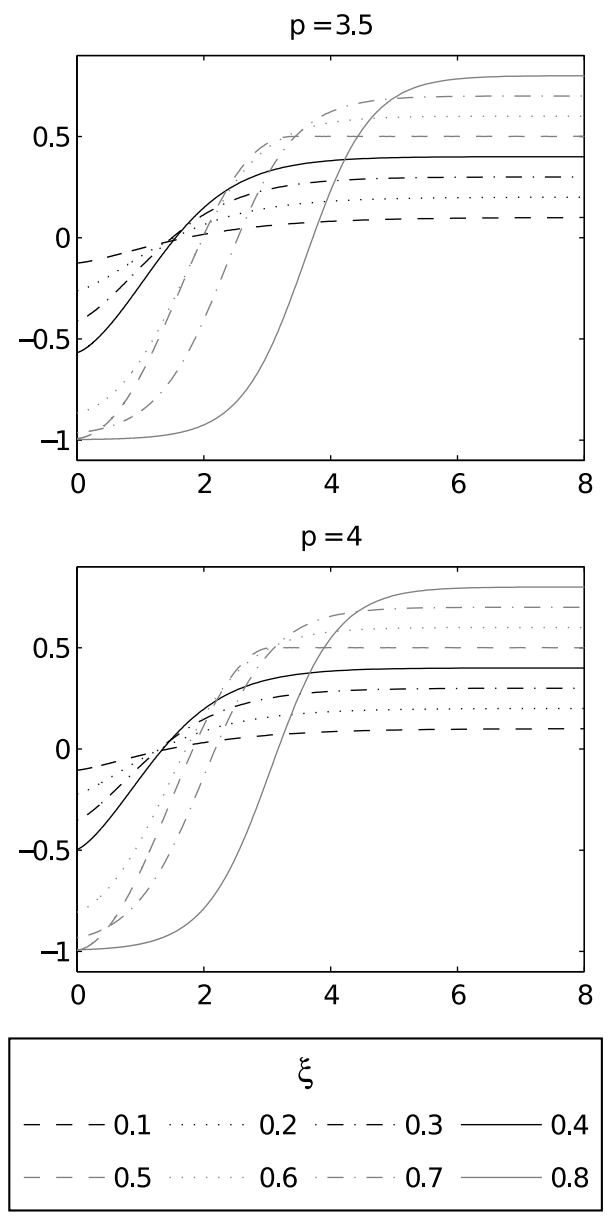

Figure 3: Solution graphs, $\rho(r)$, for different values of $p$. 
Figure 4: The dependence of the bubble radius $R$ on $\xi$, for $p=2$ (bright gray), $p=3$ (black), and $p=4$ (dark gray).

Figure 5: The dependence of $\rho_{0}$ on $\xi$, for $p=2$ (bright gray), $p=3$ (black), and $p=4$ (dark gray).

$\xi \rightarrow 1$, reaching its minimal value for $\xi \approx 0.28$. According to Figure 4 , similar behavior of the bubble radius is observed for larger values of $p$. On the other hand, for all considered values of $p$, the density $\rho_{0}$ tends to -1 for $\xi \rightarrow 1$, cf. Figure 5 .

\section{Conclusions}

A generalization of the density profile equation for nonhomogeneous fluids has been analyzed. Here, the classical Laplacian was replaced by a degenerate one. The right-hand side of the considered differential equation has been defined in such a way that the BVP under consideration has at least one strictly monotone solution. Numerical simulations suggest that some of the main properties of the solution known from the case $p=2$, are also preserved for other values of $p, 2<p<4$.

In the present implementation, the shooting method provides good approximations for $p \in[2,4]$ and $\xi \in[0.2,0.7]$, except for $p=4, \xi=0.2$. For values of $\xi$ smaller than 0.2 or greater than 0.7 , it is difficult to find the correct values of the shooting parameter $\rho_{0}$, because small changes of this parameter often result in a blow-up or a nonmonotone solution.

For the collocation method, the $p$-Laplacian case is more difficult to handle than the case $p=2$. This follows from the fact that for $p>2$ the second and higher derivatives of the solution become unbounded at the origin which makes the adaptive mesh selection inapplicable. Moreover, for values of $p$ in the range $2<p<3$ the condition numbers of the matrices involved in the Newton iteration become very large and consequently, numerical approximations become less accurate. On the other hand, the collocation method shows a very good performance for moderate values of $\xi$. 
A different variant of the shooting method has been implemented in [21], where the approximate solutions of the involved initial value problems were obtained from a different solver. In the future, we plan to apply a smoothing variable substitution, resulting in moderate higher solution derivatives near the origin which in turn shall improve the performance of the collocation method.

\section{References}

[1] W. Auzinger, G. Kneisl, O. Koch, And E. Weinmüller, A collocation code for boundary value problems in ordinary differential equations, Numer. Algorithms, 33, 27-39 (2003).

[2] W. Auzinger, O. Koch, D. Praetorius, and E. Weinmüller, New a posteriori error estimates for singular boundary value problems, Numer. Algorithms, 40, 79-100 (2005).

[3] W. Auzinger, O. Koch, And E. Weinmüller, Analysis of a new error estimate for collocation methods applied to singular boundary value problems, SIAM J. Numer. Anal., 42, 2366-2386 (2005).

[4] C.J. Budd, O. КосH, ANd E. Weinmüller, Computation of self-similar solution profiles for the nonlinear Schrödinger equation, Computing, 77, 335-346 (2006).

[5] J. W. CAhn And J. E. Hilliard, Free energy of a nonuniform system. I. Interfacial free enrgy,J. Chem Phys., 28, 258-267 (1958).

[6] F. Dell'Isola, H. Gouin AND G. Rotoli, Nucleation and shell-like interfaces by second-gradient theory: numerical simulations, Eur. J. Mech. B/Fluids, 15, 545-568 (1996).

[7] F. Dell'Isola, H. Gouin AND P. Seppecher Radius and surface tension of microscopic bubbles second-gradient theory,C.R. Acad. Sci. Paris, 320 (serie IIb), 211-216 (1995)

[8] P. Drábek, R.F. Manásevich, And P. TAKÁc, Manifolds of critical points in a quasi-linear model for phase transitions, Contemporary Mathematics, 540, 95-134 (2011).

[9] B. Franchi, E. LANCONElli, AND J. SERrin, Existence and uniqueness of nonnegative solutions of quasilinear equations in $R^{n}$, Adv. Math., 118, 177-243 (1996).

[10] M.E. Gurtin, D. Polignone, And J. Vinals, Two-phase binary fluids and immiscible fluids described by an order parameter, Math. Models Methods Appl. Sci., 6, 815-831 (1996).

[11] F.R. DE Hoog AND R. WeIss, Difference Methods for Boundary Value Problems with a Singularity of the First Kind, SIAM J. Numer. Anal., 13, 775-813 (1976).

[12] F.R. De Hoog And R. Weiss, Collocation Methods for Singular Boundary Value Problems, SIAM J. Numer. Anal., 15, 198-217 (1978).

[13] F.R. DE Hoog AND R. Weiss, On the Boundary Value Problem for Systems of Ordinary Differential Equations with a Singularity of the Second Kind, SIAM J. Math. Anal., 11, 41-60 (1980).

[14] G. Kitzhofer, O. Koch, P.M. Lima, AND E. Weinmuller, Efficient numerical solution of the density profile equation in hydrodynamics, J. Sci. Comput., 32, 411-424 (2007).

[15] G. Kitzhofer, O. Koch, G. Pulverer, Ch. Simon, and E. Weinmüller, The new Matlab code bvpsuite for the solution of singular implicit BVPs, J. Numer. Anal. Indust. Appl. Math., 5, 113-134 (2010).

[16] G. Kitzhofer, Numerical Treatment of Implicit Singular BVPs, Ph.D. Thesis, Inst. for Anal. and Sci. Comput., Vienna Univ. of Technology, Austria, 2013.

[17] G. Kitzhofer, O. Kосh, And E. Weinmüller, Pathfollowing for essentially singular boundary value problems with application to the complex Ginzburg-Landau equation, BIT, 49, 217-245 (2009).

[18] О. KосH, Asymptotically correct error estimation for collocation methods applied to singular boundary value problems, Numer. Math., 101, 143-164 (2005).

[19] O. Koch, R. März, D. Praetorius, and E.B. Weinmüller, Collocation methods for index-1 DAEs with a singularity of the first kind, Math. Comp., 79, 281-304 (2010).

[20] N.B. Konyukhova, P.M. Lima, M.L. Morgado, And M.B. Soloviev, Bubbles and droplets in nonlinear physics models: Analysis and numerical simulation of singular nonlinear boundary value problems, Comp. Maths. Math. Phys., 48, 20182058 (2008).

[21] G. YU. Kulikov,P.M. Lima, And M. L. Morgado, Analysis and numerical approximation of singular boundary value problems with $p$-Laplacians in fluid mechanics, submitted.

[22] P.M. Lima, N.B. Konyukhova, N.V. Chemetov, And A.I. Sukov, Analytical-numerical investigation of bubble-type solutions of nonlinear singular problems, J. Comput. Appl. Math., 189, 260-273 (2006).

[23] G. Pulverer, G. Söderlind, And E. Weinmüller, Automatic grid control in adaptive BVP solvers, Numer. Algorithms, $56,61-92$ (2011).

[24] V. Savin, E. Valdinoci, And B. Sciunzi, Flat level set of p-Laplace phase transitions, Mem. Amer. Math. Soc., 182 Issue 858 (2006).

[25] B. Sciunzi And E. VAldinoci, Mean curvature properties for p-Laplace phase transitions,J. Eur. Math. Soc., 7, 319-359 (2005).

[26] P. SePpecher, Moving contact lines in the Cahn-Hilliard theory, Internat. J. Engrg. Sci., 34, 997-992 (1996).

[27] P. TAKAC,Stationary readial solutions for a quasilinear Cahn-Hilliard model in $N$ space dimensions, Electr. J. Diff. Eq., Conf. 17, 227-254 (2009).

[28] S. Wolfram, Wolfram Mathematica Documentation Centre, available at http://reference.wolfram.com/mathematica/ref/NDSolve.html?q=NDSolve\&lang=en 